\title{
P2Y12 Polymorphisms and the Risk of Adverse Clinical Events in Patients Treated with Clopidogrel: A Meta-Analysis
}

\section{Authors}

Kun Zhao ${ }^{1,2^{*}}$, Ming Yang ${ }^{3^{*}}$, Yanxia $\mathrm{Lu}^{4}$, Shusen Sun ${ }^{5}$, Wei Li ${ }^{6}$, Xingang $\mathrm{Li}^{1,6}$, Zhigang Zhao ${ }^{1,6}$

\section{Affiliations}

1 Department of Pharmacy, Beijing Tiantan Hospital, Capital Medical University, Beijing, People's Republic of China

2 Department of Pharmacy, Beijing Friendship Hospital Pinggu Campus, Capital Medical University, Beijing, People's Republic of China

3 Department of Anesthesiology, Tianjin Eye Hospital, Tianjin, People's Republic of China

4 Department of Pharmacy, The General Hospital of the Chinese People's Armed Police Forces, Beijing, People's Republic of China

5 College of Pharmacy and Health Sciences, Western New England University, Springfield, Massachusetts, United States of America

6 Monogenic Disease Research Center for Neurological Disorders, Beijing Tiantan Hospital, Capital Medical University, Beijing, People's Republic of China

\section{Key words}

Clopidogrel, adverse clinical events, P2Y12 polymorphism, meta-analysis

received 24.02 .2018

accepted 25.04.2018

\section{Bibliography}

DOI https://doi.org/10.1055/a-0622-8110

Published online: 23.5 .2018

Drug Res 2019; 69: 23-31

(c) Georg Thieme Verlag KG Stuttgart · New York

ISSN 2194-9379

\section{Correspondence}

Xingang Li

Department of Pharmacy

Beijing Tiantan Hospital

Capital Medical University

No. 6 TiantanXili, Dongcheng District

100050 Beijing

China

Tel.: + 86/010/67096 857, Fax: + 86/010/67096867

lxg198320022003@163.com

* Kun Zhao and Ming Yang contributed equally to this work and should be considered as co-first authors.

\author{
Zhigang Zhao \\ Department of Pharmacy \\ Beijing Tiantan Hospital \\ Capital Medical University \\ No. 6 TiantanXili, Dongcheng District \\ 100050 Beijing \\ China \\ Tel.: + 86/010/67098 036, Fax: + 86/010/67096 867 \\ 1022zzg@sina.com
}

Supporting Information for this article is available online at http://www.thieme-connect.de/products

\section{ABSTRACT}

Background and study aim Some studies have reported an association between P2Y12 gene polymorphisms and clopidogrel adverse outcomes with inconsistent results. We aimed to explore the relationship between $\mathrm{P} 2 \mathrm{Y} 12$ polymorphisms and the risk of adverse clinical events in patients treated with clopidogrel through a meta-analysis.

Methods A systematic search of PubMed, Web of Science and the Cochrane Library was conducted. Retrieved articles were comprehensively reviewed and eligible studies were included, and the relevant data was extracted for this meta-analysis. All statistical tests were performed by the Review Manager 5.3 software.

Results A total of 14 studies involving 8,698 patients were included. In the Han Chinese population, ischemic events were associated with $\mathrm{P} 2 \mathrm{Y} 12 \mathrm{~T} 744 \mathrm{C}$ polymorphism in the CC vs $\mathrm{TT}+\mathrm{CT}$ genetic model $(\mathrm{OR}=3.32,95 \% \mathrm{Cl}=1.62-6.82$, $\mathrm{P}=0.001)$, and the events were associated with P2Y12 C34T polymorphism in the TT + TC vs CC genetic model $(\mathrm{OR}=1.70$, $95 \% \mathrm{Cl}=1.22-2.36, \mathrm{P}=0.002)$. However, ischemic events were not related to $\mathrm{P} 2 \mathrm{Y} 12 \mathrm{G} 52 \mathrm{~T}$ polymorphism (TT + TG vs GG: $\mathrm{OR}=1.13,95 \% \mathrm{Cl}=0.76-1.68, \mathrm{P}=0.56$; $\mathrm{TT}$ vs $\mathrm{GG}+\mathrm{TG}$ : $\mathrm{OR}=2.02,95 \% \mathrm{Cl}=0.65-6.28, \mathrm{P}=0.22)$. The associations between the $\mathrm{P} 2 \mathrm{Y} 12$ polymorphism and ischemic events were not significant in T744C, G52T and C34T genotype for another subgroup of the Caucasian population $(P>0.05)$. Only two studies referring to bleeding events were included in this analysis of C34T polymorphism, and no significant association was found ( $T T+T C$ vs CC: $O R=1.07,95 \% C l=0.37-3.15, P=0.90)$. Conclusions In the Caucasian population, $\mathrm{P} 2 \mathrm{Y} 12$ gene polymorphisms are not associated with clinical events. However, in the Chinese Han population, P2Y12 T744C and C34T polymorphisms are significantly associated with adverse clinical events. 


\section{Introduction}

Clopidogrel is an important antiplatelet drug, inhibiting the adenosine diphosphate (ADP)-induced platelet aggregation. Aspirin plus clopidogrel are recommended for the treatment and prevention of cerebrovascular and cardiovascular diseases[1]. Clopidogrel has been widely used for ischemic stroke (IS) and acute coronary syndrome (ACS), particularly in patients undergoing percutaneous coronary intervention (PCI)[2]. However, some patients still have adverse clinical outcomes with clopidogrel treatment, including composite ischemic events [transient ischemic attack (TIA), myocardial infarction (MI), target vessel revascularization (TVR), stent thrombosis (ST), vascular-related mortality, etc.] and bleeding events[3-5].

Genetic polymorphisms play a vital role in the clopidogrel response variability[6]. Clopidogrel inhibits the platelet P2Y12 (purinergic receptor $\mathrm{P} 2 \mathrm{Y}, \mathrm{G}$-protein coupled, 12 ) receptor, and the receptor gene polymorphisms may be an important factor in the drug response. T744C (rs2046934), G52T (rs6809699) and C34T (rs6785930) polymorphisms have been reported frequently for the association of the $\mathrm{P} 2 \mathrm{Y} 12$ receptor gene and adverse clinical events. Haplotype $(\mathrm{H} 1 / \mathrm{H} 2)$ could be generated by the C139T, T744C, ins801A and G52T polymorphisms, as the four single nucleotide polymorphisms (SNPs) are in complete linkage disequilibrium. The C34T polymorphism is not linked to the four SNPs[7].

A number of studies have reported the association of P2Y12 gene polymorphisms with clopidogrel adverse clinical events. For T744C, LiXQ considered that the T allele was associated with an increased risk of major adverse cardiac events[8]. While Sun B showed that the impact of T744C polymorphism on the recurrence of ischemic events was not significant[9]. Studies of other mutation alleles had similarly inconsistent results. This led us to conduct a meta-analysis of the published studies, and we aimed to systematically identify the association of P2Y12 gene polymorphisms with the risk of adverse clinical events in clopidogrel treated patients.

\section{Materials and Methods}

\section{Literature search}

The electronic databases of PubMed, Web of Science and the Cochrane Library were searched. Search terms were "P2RY12 OR P2Y12” AND “clopidogrel OR antiplatelet OR platelet” AND “single nucleotide polymorphisms OR SNP OR polymorphism OR variant OR variation". All eligible studies were retrieved by full texts on March 23, 2018. The bibliographies of the included articles were searched to identify other pertinent articles.

\section{Inclusion and exclusion criteria}

The inclusion criteria were: (1) published in English, (2) case-control studies, (3) patients with cardiovascular or cerebrovascular diseases, (4) patients receiving a maintenance dose $75 \mathrm{mg} /$ day of clopidogrel and (5) with complete data about P2Y12 genotyping and adverse clinical events. Animal trials, case reports, reviews, studies with incomplete data about P2Y12 gene and adverse clinical events, conference papers, and studies with a follow-up $<3$ months were excluded.

\section{Data extraction}

Two reviewers independently screened all retrieved studies by titles and abstracts, and then by the full texts. Eligible studies were obtained by using the inclusion and exclusion criteria. Any disagreements were resolved by discussion. Standardized forms were used for data extraction, including the first author of a study, publication year, country, race, studied population, treatment protocol, sample size, P2Y12 genotype, follow-up time, and all adverse clinical outcomes. The Newcastle-Ottawa Scale (NOS) was used for assessing the quality of the included studies[10].

\section{Statistical analysis}

All statistical tests for this meta-analysis were performed by the Review Manager 5.3 (The Cochrane Collaboration, Oxford, UK). The odds ratio (OR) with $95 \%$ confidence interval $(\mathrm{Cl})$ was used to assess the strength of the association between $\mathrm{P} 2 \mathrm{Y} 12$ polymorphisms and adverse clinical events in clopidogrel-treated patients. The test for heterogeneity was performed for each meta-analysis. The fixedeffects model (FE) was adopted if there was no statistical difference $\left(P>0.1, I^{2}<50 \%\right)$, otherwise the random-effects model (RE) was chosen[11]. Sensitivity analyses were conducted by an individual exclusion of each study to assess its effect on the pooled outcome OR[12]. The publication bias was assessed by the funnel plot. $\mathrm{P}<0.05$ indicates a significant difference among different genotypes[13].

\section{Results}

\section{Study characteristics}

A total of 668 articles were identified through database searching, of which 210 duplicate articles were excluded. Additionally, 434 articles (238 irrelevant studies, 119 reviews, 8 conference papers, 59 studies without adverse events, 4 animal trials, 4 case reports, and 2 non-English-language study) were excluded by title and abstract screening. Then, six studies[14-18] with incomplete data, one study[19] with incorrect data, one study[20] with a followup $<3$ months and one study[21] without full-text were excluded respectively. Fifteen studies [8, 9, 22-34] met the inclusion criteria. However, one study[26] was not included, because the genotypes of all recruited population were of homozygous wild-type (GG). A total of 14 studies were included in this meta-analysis (Supporting Material 1), including 8,698 patients. All patients received aspirin in these studies with pre-specified ischemic or bleeding events. The NOS value of each article is greater than 5 . The baseline characteristics (race, patient population, treatment protocol, sample size, genetic locus, follow-up period, outcomes, NOS score etc.) of the studies are presented in the Supporting Material 2.

\section{Meta-analysis results}

Association of the P2Y12 T744C polymorphism with ischemic events.

Eight of the fourteen included studies reported an association between the P2Y12 T744C polymorphism and adverse clinical events in clopidogrel-treated patients. In the CC + CT vs TT genetic model, a total of 4,348 patients were enrolled: 435 had ischemic events and 1,329 were carriers of the $C$ allele. In another genetic model (CC vs 


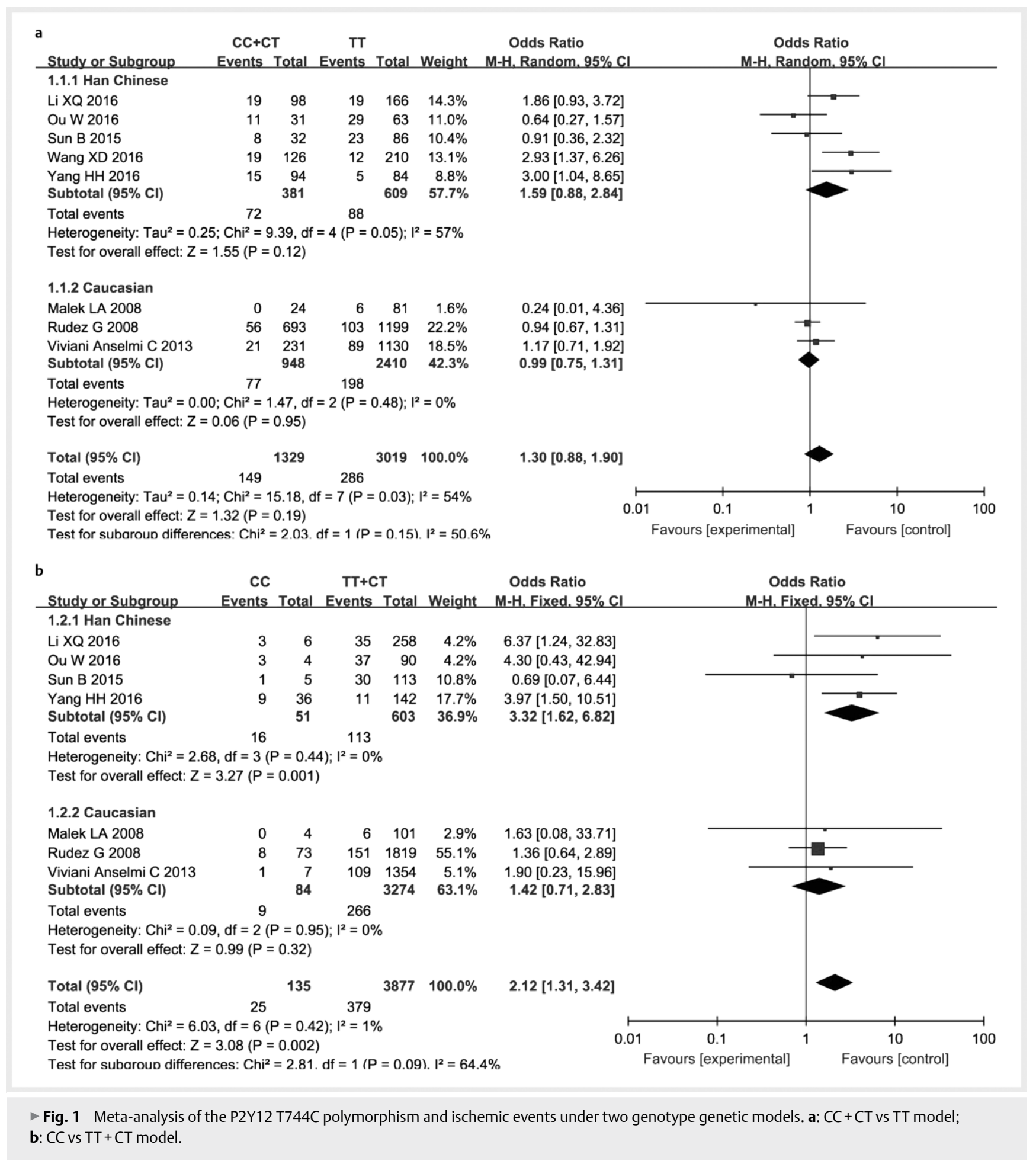

$\mathrm{TT}+\mathrm{CT}$ ), seven studies including 4,012 patients were analyzed. The heterogeneity test was performed in two different genotype genetic models (CC + CT vs TT: $P=0.03, \mathrm{I}^{2}=54 \%$ and CC vs TT $+C T: P=0.42$, $\mathrm{I}^{2}=1 \%$ ), and the random-effects model and the fixed-effects model was respectively used for this analysis ( $\triangleright$ Fig. 1 ). In the analysis of CC + CT vs TT genetic model, no significant association was found between the P2Y12 T744C polymorphism and ischemic events either in the Han Chinese population $(\mathrm{OR}=1.59,95 \% \mathrm{Cl}=0.88-2.84$,
$\mathrm{P}=0.12)$ or in the Caucasian population $(\mathrm{OR}=0.99,95 \% \mathrm{Cl}=0.75$ 1.31, $\mathrm{P}=0.95)$. However, in another analysis of CC vs TT + CT genetic model, the correlation between the $\mathrm{P} 2 \mathrm{Y} 12 \mathrm{~T} 744 \mathrm{C}$ polymorphism and ischemic events was significant in the Han Chinese population $(\mathrm{OR}=3.32,95 \% \mathrm{Cl}=1.62-6.82, \mathrm{P}=0.001)$, but it was not significant in the Caucasian population $(\mathrm{OR}=1.42,95 \% \mathrm{Cl}=0.71-2.83$, $\mathrm{P}=0.32$ ). 


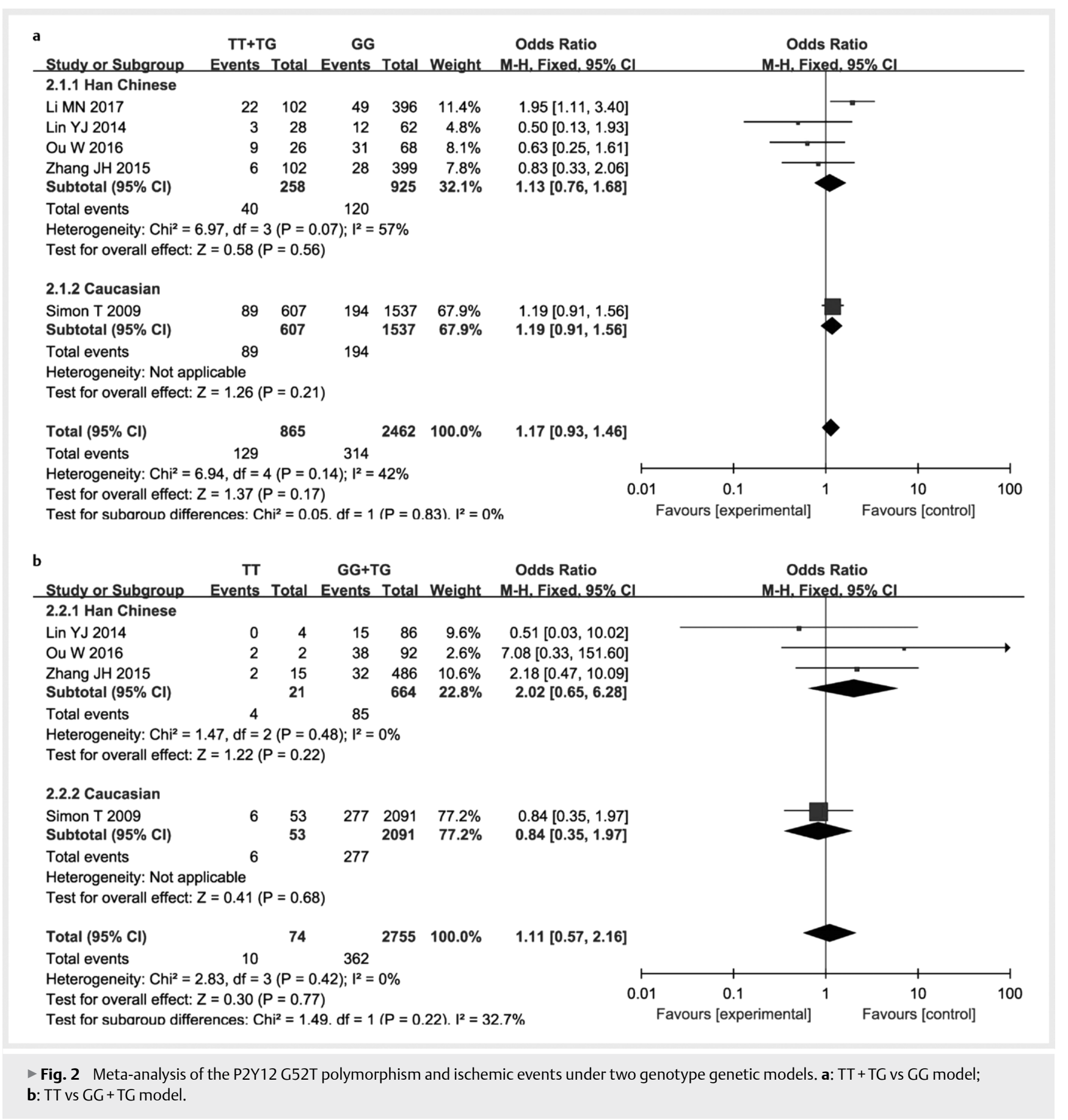

Association of the P2Y12 G52T polymorphism with ischemic events.

Five studies reported an association of the P2Y12 G52T polymorphism with adverse clinical outcomes. Only one study was conducted in the Caucasian population, and the other four studies were in the Han Chinese population. Of the 3327 subjects, 443 had ischemic events, and 865 were carriers of the T allele in the TT + TG vs GG genetic model. However, four studies with complete data were enrolled in another genetic model, including a total of 2,829 subjects. The fixed-effects model was performed because a lower heterogeneity was identified $\left(\mathrm{I}^{2}=42 \%\right.$ and $\left.\mathrm{I}^{2}=0 \%\right)$ in different genotype ge- netic models ( $\triangleright$ Fig. 2 ). The analysis indicated that ischemic events were not associated with the P2Y12 G52T polymorphism (TT + TG vs $\mathrm{GG}: \mathrm{OR}=1.13,95 \% \mathrm{Cl}=0.76-1.68, \mathrm{P}=0.56$; $\mathrm{TT}$ vs $\mathrm{GG}+\mathrm{TG}: \mathrm{OR}=2.02$, $95 \% \mathrm{Cl}=0.65-6.28, \mathrm{P}=0.22)$ in the Han Chinese population.

Association of the P2Y12 C34T polymorphism with ischemic events

Seven studies reporting an association of the P2Y12 C34T polymorphism with ischemic events were included. A total of 4,176 patients were recruited in the TT + TC vs CC genetic model, while only four studies provided complete information in the TT vs CC + TC genet- 


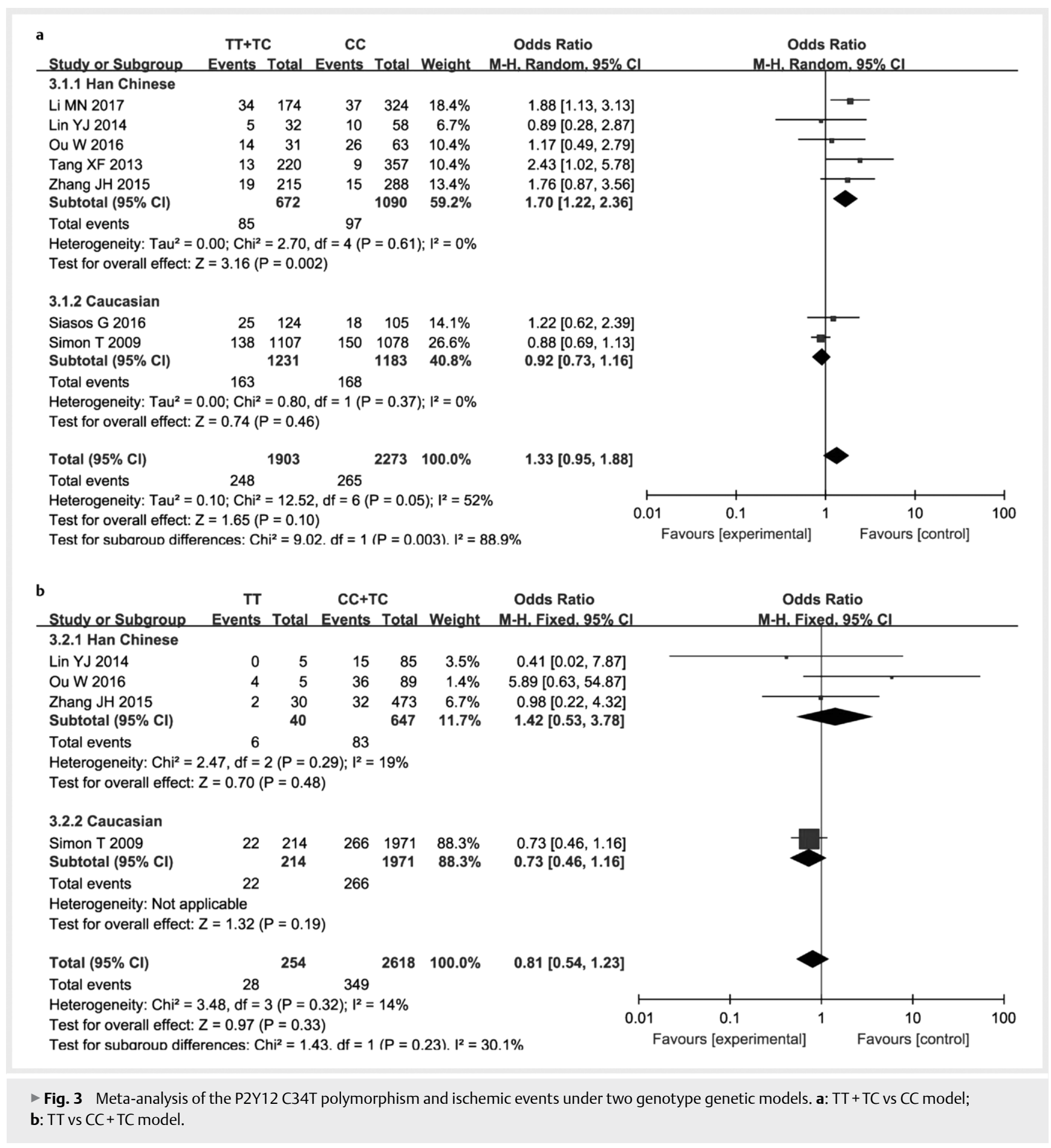

ic model. The fixed-effects model and the random-effects model were adopted respectively after heterogeneity testing ( $\vee$ Fig. 3 ). In the Han Chinese population, a significant association between the P2Y12 C34T polymorphism and ischemic events was found under the TT + TC vs CC genetic model $(O R=1.70,95 \% C l=1.22$ $2.36, \mathrm{P}=0.002)$. However, the association was not found in the Caucasian population $(\mathrm{OR}=0.92,95 \% \mathrm{Cl}=0.73-1.16, \mathrm{P}=0.46)$. Meanwhile, the P2Y12 C34T polymorphism was unrelated to ischemic events under the TT vs CC + TC genetic model in both populations.
Association of the P2Y12 C34T polymorphism with bleeding events

Two studies referring to bleeding events were included in the analysis of the C34T polymorphism. The association of the P2Y12 C34T polymorphism with bleeding events was analyzed under the TT + TC vs CC genetic model. The random-effects model was chosen on the basis of a higher heterogeneity $\left(I^{2}=82 \%\right.$, > Fig. 4). No significant association was found in this analysis $(\mathrm{OR}=1.07,95 \% \mathrm{Cl}=0.37-3.15$, $\mathrm{P}=0.90)$. 


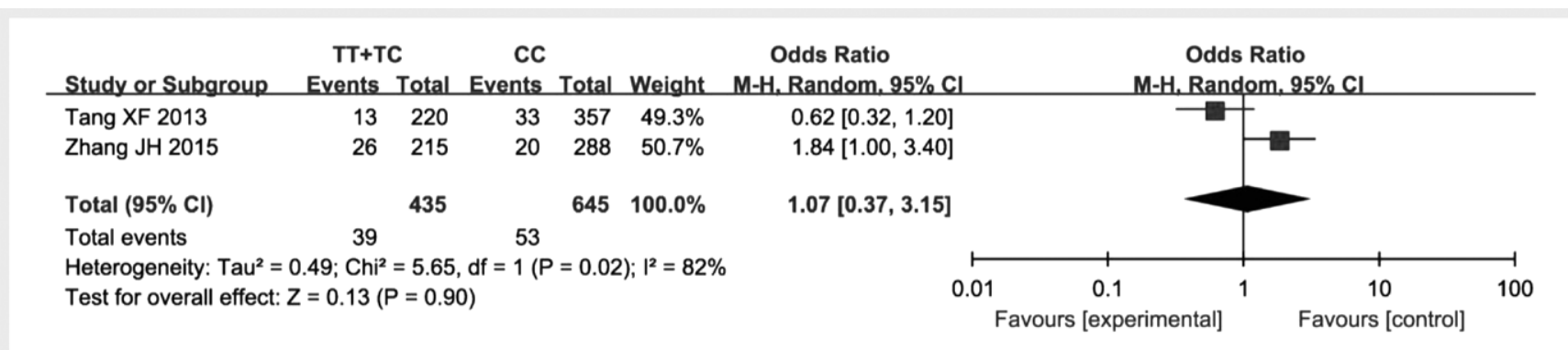

Fig. 4 Meta-analysis of the P2Y12 C34T polymorphism and bleeding events under the TT + TC vs CC model.

- Table 1 Sensitivity analysis of the P2Y12 T744C polymorphism and ischemic events in the Han Chinese population.

\begin{tabular}{|c|c|c|c|}
\hline \multicolumn{2}{|c|}{ CC+ CT vs TT } & \multicolumn{2}{c|}{ CC vs TT+ CT } \\
\hline $\begin{array}{c}\text { Study } \\
\text { Exclusion }\end{array}$ & $\begin{array}{c}\text { RE } \\
(\boldsymbol{P} \text { value })\end{array}$ & $\begin{array}{c}\text { Study } \\
\text { Exclusion }\end{array}$ & $\begin{array}{c}\text { FE } \\
(\boldsymbol{P} \text { value })\end{array}$ \\
\hline Li XQ 2016 & 0.31 & Li XQ 2016 & 0.008 \\
\hline Ou W 2016 & 0.008 & Ou W 2016 & 0.003 \\
\hline Sun B 2015 & 0.08 & Sun B 2015 & 0.0003 \\
\hline Wang XD 2016 & 0.38 & / & $/$ \\
\hline Yang HH 2016 & 0.32 & Yang HH 2016 & 0.07 \\
\hline FE: fixed-effects model, RE: random-effects model. \\
\hline
\end{tabular}

\section{Sensitivity analysis}

Sensitivity analysis was performed by the exclusion of a single study in turn. In this analysis of the Han Chinese population on the P2Y12 T744C polymorphism, the pooled P-values changed respectively when Ou W[33] was excluded under the CC + CT vs TT genetic model and when Yang $\mathrm{HH}$ [32] was excluded under the CC vs TT + CT genetic model. However, no other single study could influence the pooled $\mathrm{P}$-values in the remaining exclusion analyses ( $\triangleright$ Table $\mathbf{1}$ ).

\section{Publication bias}

The publication bias was evaluated by funnel plots, and the shapes were symmetrical, suggesting no publication bias among these analyses (Supporting Material 3). The funnel plot of bleeding events was not performed because only two studies were included.

\section{Discussion}

Clopidogrel is a thienopyridine derivative inhibiting ADP-mediated platelet aggregation[35]. The active metabolite binds irreversibly and specifically to the P2Y12 platelet receptor. An individual variability is observed in patients receiving clopidogrel treatment, and some patients can experience adverse clinical events[4]. Clopidogrel poor response is likely caused by multiple factors, including genetics, increasing age, greater body mass index (BMI), higher triglyceride levels and lower levels of high-density lipoprotein cholesterol[36, 37].

The P2Y12 gene is located in the q25. 1 region of chromosome 3, and it belongs to the $\mathrm{G}$-protein coupled receptor family. The coupling of the P2Y12 protein with the inhibitory $G$ alpha protein subunit results in the inhibition of cyclic adenosine monophosphate (cAMP) production, and therefore a subsequent reduction in the intracellular cAMP content. Furthermore, P2Y12 receptor induces a platelet aggregation through a weak activation of glycoprotein IIb/IIla integrin via the phosphoinositide kinase-3 pathway [7]. Platelet activation by ADP plays a crucial role in hemostasis and thrombosis. Numerous studies have established the key role of this receptor in the ADP-dependent amplification of platelet aggregation induced by other agonists, such as thromboxane $A 2$ and thrombin $[38,39]$. The antithrombotic effect of clopidogrel and the ADP-induced platelet response are considerably variable. Therefore, the P2Y12 gene is screened for possible variants.

Many studies investigated the relationship of the P2Y12 gene polymorphism and adverse clinical events, but these studies $[8,9,22-34]$ had divergent results. It is therefore necessary to perform a meta-analysis exploring this association. A total of 14 articles were included, and the NOS value of each article is greater than 5. Three genotypes (T744C, G52T and C34T) of the P2Y12 gene were analyzed in our study.

T744C is located at the 744 nucleotide after the $5^{\prime}$ intron start site and consists of a T-to-C transition [7]. Both C34T and G52T are located in the coding region of the P2Y12 gene (cds-synon). Fontana $\mathrm{P}$ et al. examined ADP-induced platelet aggregation responses in healthy volunteers, and sequence variations in the P2Y12 gene were investigated. They identified two phenotypic groups (haplotypes $\mathrm{H} 1$ and $\mathrm{H} 2$ ) of subjects with high and low responsiveness to 2 micromol/L ADP [7]. Four frequent polymorphisms (i-C139T (rs 10935838), i-T744C (rs2046934), i-ins801A (rs5853517), G52T (rs6809699)) were in total linkage disequilibrium, determining haplotypes $\mathrm{H} 1$ and $\mathrm{H} 2$, with respective allelic frequencies of 0.86 and 0.14 . The presence of the minor haplotype $\mathrm{H} 2$ was associated with an enhanced ADP-induced platelet aggregation in healthy volunteers. The molecular mechanism by which the $\mathrm{H} 2$ haplotype increases ADP-induced platelet aggregation remains unclear. Based on the investigated sequence, an amino acid substitution affecting the protein structure or splice variants could be ruled out. Thus, an increase in the number of platelet surface receptors is most likely the explanation [7]. C34T is not part of the haplotype and no function research was found until now.

Based on the analysis of subgroups, significant associations between the P2Y12 T744C/C34T polymorphism and ischemic events were found $(P<0.05)$, but the association of the P2Y12 G52T polymorphism and ischemic events is not observed $(P>0.05)$ in the Han Chinese population. Ischemic events are related to the P2Y12 T744C polymorphism under the CC vs TT + CT genetic model $(P=0.001)$, therefore the $T 744 C$ genotype may predict the risk of 
recurrent ischemic events in the Han Chinese patients. Patients with a mutant homozygote (CC) of the T744C genotype had a higher risk of recurrent ischemic events within one year $(O R>1)$. A total of $7.8 \%$ of all enrolled patients were mutant homozygote, and $31.4 \%$ of them had recurrent ischemic events. As for the sensitivity analysis, the pooled $\mathrm{P}$ values changed when the Ou W[33] study was excluded in the $C C+C T$ vs TT genetic model $(P=0.008)$, and when Yang $\mathrm{HH}$ [32] was excluded in the CC vs TT $+\mathrm{CT}$ genetic model $(P=0.07)$. Ou W suggested that T744C variants were not associated with a response to clopidogrel (weight $11.0 \%$ ), and the incidence of ischemic events was not different between the mutant $C$ allele carriers and non-carriers. Yang $\mathrm{HH}$ found that the T744C polymorphism was associated with the efficacy of clopidogrel treatment (i. e. reduction of major adverse cardiac events), and patients carrying the CC genotype were more likely to have ischemic events than other genotypes. Second, ischemic events and the P2Y12 C34T polymorphism are related under the TT + TC vs CC genetic model in the Han Chinese population. P2Y12 C34T polymorphisms may predict the risk of recurrent ischemic events, and carriers of the mutant T allele have a higher risk of composite ischemic events after taking clopidogrel. A total of $38.1 \%$ of patients were carriers of the mutant T allele, and $12.6 \%$ of them had recurrent ischemic events compared to $8.9 \%$ of the non-carriers. According to the sensitivity analysis, the pooled P-values are not significantly affected when each single study is excluded respectively. The results were similar in sensitivity analyses for studies between G52T polymorphism and clinical events. Sionova $M$ et al. investigated the association of P2Y12 polymorphisms (C34T and T744C) with increased risk of peri-procedural bleeding in patients undergoing coronary angiography/PCI. No significant association of the SNPs of P2Y12 C32T and T744C with an increased risk of peri-procedural bleeding was found in their patients[40]. Since the study had a short-term follow-up (30 days), it was excluded from the meta-analysis.

In addition, studies on the P2Y12 gene polymorphism and the incidence of ischemic events in the Caucasian population failed to establish an association. The pooled P-value was not influenced when excluding any single study, revealing that a single study could not change the result of the Caucasian population analysis.

After data on the Han Chinese and the Caucasian populations were merged, there was no significant correlation between the three locus variants and clinical events (ischemia and hemorrhage) after drug administration. However, the subgroup analysis found clinical events were associated with genetic variants (T744C and C34T) in the Han Chinese population but not in Caucasians. We speculate the reasons are as follows: 1 ) the studies included in this analysis are mostly based on the data within the Chinese population, and there are few reports on the Caucasian population. The smaller sample size of the Caucasian population may lead to biased results; 2 ) there is a large difference in the frequency of gene mutations between races. The mutation rate of T744C in Chinese population is $16.0 \%-30.6 \%$, versus $8.7 \%-19.9 \%$ in the Caucasian population. The mutation frequency of $\mathrm{C} 34 \mathrm{~T}$ in the Chinese population is $20.5 \%-21.2 \%$, versus around $43.3 \%$ in the Caucasian population. The differences in sample size and the different mutation frequencies between races may lead to inconsistencies in study results.

Several limitations in this meta-analysis should be noted: 1 ) the sample size in these included studies was relatively small, which may reduce the statistical power, 2) lack of original data in published studies limited further analysis, 3) the majority of the included studies were conducted in Han Chinese patients and Caucasian patients, and further studies are needed from different ethnic groups, and 4) due to inadequate data, certain ischemic events were not predicted, such as TIA, MI, TVR, ST, and other fatal vascular-related events.

\section{Conclusions}

The P2Y12 T744C and C34T polymorphisms are found to be associated with ischemic events in Han Chinese patients treated with clopidogrel, and the P2Y12 G52T polymorphism has no significant impact on ischemic events. Patients with the CC homozygote of the T744C genotype have a higher risk of recurrent ischemic events, and the carriers of the mutant T allele of the C34T genotype are more likely than non-carriers to have ischemic events. However, the risk of clinical ischemic events is not related to P2Y12 T744C, G52T and C34T polymorphisms in the Caucasian population. Bleeding events are not significantly associated with the P2Y12 C34T polymorphism in the Han Chinese population. Further research on the relationship of other genetic factors of P2Y12 and the risks of bleeding events in patients treated with clopidogrel is needed.

\section{Authors' contribution}

$X \mathrm{Li}$ and Z Zhao conceived and designed the study. K Zhao, Ming Yang and $S$ Sun performed the analysis and wrote the manuscript. Y Lu, W Li and Z Zhao supervised the quality of the study. All authors read and approved the final manuscript.

\section{Acknowledgements}

This work was supported by the National Natural Science Foundation of China [81503157] and the Beijing Municipal Administration of Hospital Mission Plan [QML20170506]. We also thank all the participants in this study.

\section{Conflict of Interest}

No conflict of interest has been declared by the author(s).

\section{References}

[1] Tang M, Mukundan M, Yang J et al. Antiplatelet agents aspirin and clopidogrel are hydrolyzed by distinct carboxylesterases, and clopidogrel is transesterificated in the presence of ethyl alcohol. J Pharmacol Exp Ther 2006; 319: 1467-1476

[2] Wright RS, Anderson JL, Adams CD et al. 2011; ACCF/AHA Focused Update of the Guidelines for the Management of Patients With Unstable Angina/ Non-ST-Elevation Myocardial Infarction (Updating the 2007 Guideline): A report of the American College of Cardiology Foundation/American Heart Association Task Force on Practice Guidelines. Circulation 2011; 123: 2022-2060 
[3] Qiu LN, Sun Y, Wang L et al. Influence of CYP2C19 polymorphisms on platelet reactivity and clinical outcomes in ischemic stroke patients treated with clopidogrel. Eur J Pharmacol 2015; 747: 29-35

[4] Yang J, Wang X, Peng L et al. CYP2C19 LOF alleles confer no risk for HTPR but higher risk for recurrent ischemic events in clopidogrel treated elderly ACS patients. Int J Cardiol 2015; 189: 225-227

[5] Bundhun PK, Qin T, Chen MH. Comparing the effectiveness and safety between triple antiplatelet therapy and dual antiplatelet therapy in type 2 diabetes mellitus patients after coronary stents implantation: A systematic review and meta-analysis of randomized controlled trials. BMC Cardiovasc Disord 2015; 15: 118

[6] Campo G, Fileti L, Valgimigli M et al. Poor response to clopidogrel: Current and future options for its management. J Thromb Thrombolysis 2010 ; 30 : $319-331$

[7] Fontana P, Dupont A, Gandrille S et al. Adenosine diphosphateinduced platelet aggregation is associated with $\mathrm{P} 2 \mathrm{Y} 12$ gene sequence variations in healthy subjects. Circulation 2003; 108: 989-995

[8] Li XQ, Ma N, LiXG et al. Association of PON1, P2Y12 and COX1 with Recurrent Ischemic Events in Patients with Extracranial or Intracranial Stenting. PLoS One 2016; 11: e0148891

[9] Sun B, Li ], Dong M et al. Diversity of platelet function and genetic polymorphism in clopidogrel-treated Chinese patients. Genet Mol Res 2015; 14: 1434-1442

[10] Stang A. Critical evaluation of the Newcastle-Ottawa scale for the assessment of the quality of nonrandomized studies in meta-analyses. Eur J Epidemiol 2010; 25: 603-605

[11] Su J, Xu J, Li X et al. ABCB1 C3435T polymorphism and response to clopidogrel treatment in coronary artery disease (CAD) patients: A meta-analysis. PLoS One 2012; 7: e46366

[12] Serbin MA, Guzauskas GF, Veenstra DL. Clopidogrel-Proton Pump Inhibitor Drug-Drug Interaction and Risk of Adverse Clinical Outcomes Among PCl-Treated ACS Patients: A Meta-analysis. J Manag Care Spec Pharm 2016; 22: 939-947

[13] Wen Y, Huang Z, Zhang X et al. Correlation between PON1 gene polymorphisms and breast cancer risk: A Meta-analysis. Int J. Clin Exp Med 2015; 8: 20343-20348

[14] Ziegler S, Schillinger M, Funk M et al. Association of a functional polymorphism in the clopidogrel target receptor gene, $\mathrm{P} 2 \mathrm{Y} 12$, and the risk for ischemic cerebrovascular events in patients with peripheral artery disease. Stroke 2005; 36: 1394-1399

[15] Wu H, Qian J, Xu J et al. Effects of CYP2C19 variant alleles on postclopidogrel platelet reactivity and clinical outcomes in an actual clinical setting in China. Pharmacogenet Genomics 2012; 22: 887-890

[16] Liang ZY, Han YL, Zhang XL et al. The impact of gene polymorphism and high on-treatment platelet reactivity on clinical follow-up: Outcomes in patients with acute coronary syndrome after drug-eluting stent implantation. Eurolntervention 2013; 9: 316-327

[17] Yi X, Wang Y, Lin J et al. Interaction of CYP2C19, P2Y12, and GPIIla Variants Associates With Efficacy of Clopidogrel and Adverse Events on Patients With Ischemic Stroke. Clin Appl Thromb Hemost 2016

[18] Bouman HJ, van Werkum JW, Rudez G et al. The relevance of P2Y(12)-receptor gene variation for the outcome of clopidogrel-treated patients undergoing elective coronary stent implantation: A clinical follow-up. Thromb Haemost 2012; 107: 189-191

[19] Siasos G, Oikonomou E, Vavuranakis M et al. Genotyping, Platelet Activation, and Cardiovascular Outcome in Patients after Percutaneous Coronary Intervention: Two Pieces of the Puzzle of Clopidogrel Resistance. Cardiology 2017; 137: 104-113

[20] Smith SM, Judge HM, Peters $G$ et al. Common sequence variations in the P2Y12 and CYP3A5 genes do not explain the variability in the inhibitory effects of clopidogrel therapy. Platelets 2006; 17: 250-258
[21] Li X], Chen XM. Association between clopidogrel resistance and polymorphism of platelet adenosine diphosphate receptor in patients with coronary atherosclerotic disease. Zhejiang Da Xue Xue Bao Yi Xue Ban 2014; 43: 333-338

[22] Lin Y], Li JW, Zhang MJ et al. The association between CYP2C19 genotype and of in-stent restenosis among patients with vertebral artery stent treatment. CNS Neurosci Ther 2014; 20: 125-130

[23] Malek LA, Kisiel B, Spiewak M et al. Coexisting polymorphisms of P2Y12 and CYP2C19 genes as a risk factor for persistent platelet activation with clopidogrel. Circ J 2008; 72: 1165-1169

[24] Zhang JH, Wang J, Tang XF et al. Effect of platelet receptor gene polymorphisms on outcomes in ST-elevation myocardial infarction patients after percutaneous coronary intervention. Platelets 2016; 27 : 75-79

[25] Tang XF, Zhang JH, Wang J et al. Effects of coexisting polymorphisms of CYP2C19 and P2Y12 on clopidogrel responsiveness and clinical outcome in patients with acute coronary syndromes undergoing stent-based coronary intervention. Chin Med J (Engl) 2013; 126: 1069-1075

[26] Sen HM, Silan F, Silan C et al. Effects of CYP2C19 and P2Y12 Gene Polymorphisms on Clinical Results of Patients Using Clopidogrel after Acute Ischemic Cerebrovascular Disease. Balkan J Med Genet 2014; 17 : $37-41$

[27] Simon T, Verstuyft C, Mary-Krause M et al. Genetic determinants of response to clopidogrel and cardiovascular events. N Engl J Med 2009; 360: 363-375

[28] Siasos G, Kioufis S, Oikonomou E et al. Impact of C34T P2Y12 ADP receptor polymorphism and smoking status on cardiovascular outcome in coronary artery disease patients receiving clopidogrel. Int J Cardiol 2016; 210: 161-163

[29] Rudez G, Pons D, Leebeek F et al. Platelet receptor P2RY12 haplotypes predict restenosis after percutaneous coronary interventions. Hum Mutat 2008; 29: 375-380

[30] Viviani Anselmi C, Briguori C, Roncarati R et al. Routine assessment of on-clopidogrel platelet reactivity and gene polymorphisms in predicting clinical outcome following drug-eluting stent implantation in patients with stable coronary artery disease. JACC Cardiovasc Interv 2013; 6: 1166-1175

[31] Li M, Wang H, Xuan L et al. Associations between P2RY12 gene polymorphisms and risks of clopidogrel resistance and adverse cardiovascular events after $\mathrm{PCl}$ in patients with acute coronary syndrome. Medicine (Baltimore) 2017; 96: e6553

[32] Yang HH, Chen Y, Gao CY. Associations of P2Y12R gene polymorphisms with susceptibility to coronary heart disease and clinical efficacy of antiplatelet treatment with clopidogrel. Cardiovasc Ther 2016; 34: 460-467

[33] Ou W, He Y, Li A et al. Genotype Frequencies of CYP2C19, P2Y12 and GPIIla Polymorphisms in Coronary Heart Disease Patients of Han Ethnicity, and Their Impact on Clopidogrel Responsiveness. Int Heart J 2016; 57: 586-592

[34] Wang X, Lai Y, Luo Y et al. Relationship between clopidogrel-related polymorphisms and variable platelet reactivity at 1 year: A cohort study from Han Chinese. J Res Med Sci 2016; 21: 111

[35] Li XG, Ma N, Wang B et al. The impact of P2Y12 promoter DNA methylation on the recurrence of ischemic events in Chinese patients with ischemic cerebrovascular disease. Sci Rep 2016; 6: 34570

[36] Shuldiner AR, O'Connell JR, Bliden KP et al. Association of cytochrome P450 2C19 genotype with the antiplatelet effect and clinical efficacy of clopidogrel therapy. JAMA 2009; 302: 849-857

[37] Martinelli N, Trabetti E, Pinotti M et al. Combined effect of hemostatic gene polymorphisms and the risk of myocardial infarction in patients with advanced coronary atherosclerosis. PLoS One 2008; 3: e1523 
[38] Storey RF. Biology and pharmacology of the platelet P2Y12 receptor. Curr Pharm Des 2006; 12: 1255-1259

[39] Haynes SE, Hollopeter G, Yang G et al. The P2Y12 receptor regulates microglial activation by extracellular nucleotides. Nat Neurosci 2006; 9: $1512-1519$
[40] Sionova M, Blasko P, jirous S et al. Association of polymorphisms of platelet receptors GPla $(807 \mathrm{C}>\mathrm{T}), \mathrm{GPVI}(13254 \mathrm{~T}>\mathrm{C})$, and P2Y12 (34C $>\mathrm{T}$ and $\mathrm{H} 1 / \mathrm{H} 2$ haplotype) with increased risk of periprocedural bleeding in patients undergoing coronary angiography/percutaneous coronary intervention. Postepy Kardiol Interwencyjnej 2017; 13 : 202-209 